\title{
DE L'INSTRUCTION DES COLONNES SANITAIRES VOLONTAIRES
}

Nous n'avons pas eu l'occasion de mentionner en son temps un mémoire de M. le général Dr Port dont l'importance au point de vue des secours sanitaires ne saurait être méconnue. II n'est pas inopportun d'en parler encore aujourd'hui. L'auteur, bien connu de tous les comités de la Croix-Rouge, médecin militaire de grande expérience et de grand mérife, ne craint pas de plaider la cause de l'assistance volontaire en ce qui concerne les secours sur le champ de bataille, estimant, avec raison, que l'organisation officielle est insuffisante pour satisfaire seule à cette lourde tàche en temps de guerre. Dans un précédent article il en avait déja fourni la preuve quant au transport sur le champ de bataille ${ }^{1}$, dans celui-ci il démontre qu'il en serait de même pour le service des lazarets de campagne".

L'auteur compare les ambulances volantes prussiennes, pendant la guerre de 1870, aux ambulances bavaroises. Les premières ont rendu de grands services parce qu'elles ont presque loujours été à disposition de suite, au moment d'un combat. Les secondes, par contre, avaient toujours du relard parce qu'elles avaient à Iransporter des lits. Or mieux vaut un lazaret sans lits arrivant à temps pour les premiers secours, que d'excellents lits deux ou trois jours plus tard. L'expérience a donc prouvé l'inopportunité de pourvoir les ambulances volantes de moyeus de couchage; les réquisitions et les improvisations doivent y suppléer.

Mais ce côté de la question n'est pas le plus important. C'est l'organisation du personnel qui importe le plus et il s'agit de le rendre apte à ses fonctions, non seulement au point de vue technique, comme dans une clinique correctement installée, mais aussi en vue du service spécial qu'il aura à remplir sur le champ de bataille.

A cet égard les médecins militaires se préoccupent peut-être trop peu de la question du premier pansement, tel qu'il doit être combiné pour permettre le transport immédiat de blessures graves dans les meilleures conditions possibles. Le pansement plâtré en usage ha-

1 Der Deutsche Kolonnen fuihrer, 1902, $\mathrm{n}^{\circ} 8,9,11$.

2 Id. $\mathrm{N}^{\circ} 23$ et 24. 
bituellement est parfait, sans donte, mais il ne répond absolument plus aux besoins là où la promplitude importe avant tout.

L'auteur rappelle les exigences du règlement sanitaire de l'armée allemande et constate différentes lacunes quant aux besoins actuels. Il compare les principes admis pour le transport des blessés pendant les guerres du premier Empire avtc l'état de choses qui résulte des progrès de la chirurgie moderne. Alors Larrey exigeait de son personnel sanitaire l'éloignement très rapide des blessés du théàtre des hostilités, et cela était relativement facile si l'on considère que l'amputation des membres grièvement blessés étaient alors de règle. Mais la méthode conservatrice introduite plus tard par Stromeyer a changé du tout au tout les pacédés de la chirurgie militaire moderne. La guerre sud-africaine a été le triomphe de celte chirurgie conservatrice. Or il saute aux yeux qu'elle complique singulièrement les transports, et, jusqu'ici, ni le personnel ni les règlements sanitaires ne se sont montrés à la hauteur des besoins; des réformes sont devenues urgentes surtout en ce qui concerne le transport des malades atteints de blessures graves et de fractures compliquées. A ce propos l'auteur rappelle, en passant, ce qu'il a déjà souvent proclamé et ce que l'on ne saurait assez répéter, c'est que l'usage génèreux de la morphine en injections sous-cutanées est, sur le champ de bataille, au moment du premier pansement, un adjuvant très utile du transport des blessés, en même temps qu'une mesure de charité qui s'impose.

Après avoir critiqué l'organisation sanitaire telle qu'elle existe actuellement, l'auteur tient à relever le fait que ce n'est pas l'instruction des médecins militaires qui est en défaut, mais bien les règlements sanitaires actuels qui tendent à entraver leurs services pendant les combats. Et l'auteur remarque à cette occasion que les travaux et études, de même que les progrès réalisés en ce qui concerne le transport des blessés atteints de lésions graves proviennent surtout des milieux sanitaires libres; ce qui s'explique aisément si l'on considère combien sont paralysantes toutes les formalités des organismes officiels. C'est donc du còté des sociétés de secoureurs volontaires qu'on peut espérer des résultats effectifs dans ce domaine; et c'est au fond naturel puisque une décision officielle exige normalement la mise en motvement de nombreux rouages et des lâtonnements prolongés, une adminis- 
tration ne pouvant lancer l'Etat dans des frais souvent énormes, sans que les preuves soient dûment faites de la valeur réelle du progrès à réaliser.

Rien de semblable dans les milieux libres de tonte attache offcielle; là on peut se livrer en toute liberté aux tâtonnements voulus pour viser peu à peu à la perfection rêvée; et, quand on y est parvenu, il n'y a plus à craindre encore des conflits de compétences, ou à ménager des susceptibilités. L'assistance volontaire a donc sa tâche toute marquée à cỏté de l'organisme officiel et celui-ci ne saurait avoir un avantage quelconque à reléguer ses services à l'arrière-plan et à les limiter, à la condition, naturellement, que l'assistance libre regarde comme de son premier devoir, sur les champs de bataille, de se mettre entjèrement à la disposition des autorités sanitaires militaires. Et l'auteur rappelle que c'est surtout le transport des blessures graves qui rendra cette assistance nécessaire, et que sa tâche consistera alors surtout dans l'improvisation et la fabrication rapide, sur place, d'un matérie! de transport et de pansements adapté aux besoins de différents genres de lésions en utilisant les ressources à portée.

Dans les ambulances, les fonctions des infirmiers volontaires consisteront surtout à compléter le matériel sanitaire à mesure qu'il viendra à faire défaut, et l'un des principaux services à rendre, à cet égard, au malade qu'on apporte dans l'ambulance, est de le laisser sur son brancard, aussi bien pour lui éviter un transbordement pénible que pour permettre une évacuation éventuellement rapide de tout ou partie de l'ambulance. Donc plus on fournira de brancards à un lazaret de campagne ainsi installé, mieux ce sera dans l'intérêt des malades comme dans celui du service hospitalier.

Le personnel sanitaire libre doit être instruit de telle manière qu'il puisse assurer à l'ambulance toute une variété de modes de couchage et d'articles de pansements improvisés, adaptés à chaque genre de blessure. Dans ces conditions sa présence dans un hòpital militaire ne saurait qu'être un bienfait. Il travaillera sans doute davantage avec la hache, le marteau et le ciseau qu'auprès du malade lui-même, mais qu'importe s'il y va de l'intérêt de ce dernier. Et l'auteur de compléter à cette occasion l'arsenal déjà si riche de ses procédés d'improvisation par l'indication de quelques agencements nouveaux et toujours pratiques. L'ingéniosité du général $\mathrm{D}^{\mathrm{r}}$ Port 
à cet égard est inépuisable et nous voudrions exprimer le vou qu'il nous donne bientôt une édition nouvelle et complétée de son remarquable manuel de l'improvisation.

Nous n'avons pu indiquer que sommairement le contenu de cet intéressant nıémoire. Comme on le voit le distingué médecin militaire comprend l'activité de l'assistance volontaire autrement que beaucoup de représentants des services sanitaires militaires. Evidemment là où l'assistance volontaire risque de créer une entrave quelconque à l'activité des services officiels, elle n'a que faire et doit rester au second plan, mais si l'on peut disposer d'un personnel volontaire comme lentend le $\mathrm{D}^{\mathrm{r}}$ Port, il n'y aura qu'avantage à rechercher ses services en première ligne et aucun médecin militaire conscient de sa responsabilité ne redoutera une intrusion à la fois peu gênante et précieuse pour les besoins deses blessés.

Aux sociétés de la Croix-Rouge donc de former leur personnel ainsi que l'a enseigné le Dr Port.

Dr Ferrière.

\section{DEUX BROGHURES DE M. LE $\mathrm{D}^{\mathrm{r}}$ HERMANN CRAMER}

Le transport des malades par grue.

M. le $\mathrm{D}^{\mathrm{r}} \mathrm{H}$. Gramer, à Rostock, rëdacteur de la Zeilschrift für Krankenpflege et de Der Deutsche Kolonnenführer, a fait paraître dans ce dernier périodique ${ }^{1}$ un article sur l'importance de l'emploi de la grue, non seulement pour l'embarquement des blessés, soil leur transport de la terre ferme sur les bateaux destinés à les conduire plus loin, mais même pour le chargement des malades sur les véhicules aménagés pour leur évacuation. En effet il suffit de rencontrer un arbre ou un poteau quelconque, suffisamment solide pour pouvoir installer, au moyen d'une perche oblique, de cordes, et de trois poulies un appareil qui tiendra lieu de grue et permet. tra de soulever sans effort et sans secousse les blessés jusqu'à la hauteur du véhicule qui doit les transporter. Cette installation pourra aussi servir pour hisser doucement les blessés à la hauteur

$1 \mathrm{VI}^{\circ}$ année, $\mathrm{n}^{\circ} 21$. 